\title{
Isolated complete caudate lobectomy for hepatic tumor of the anterior transhepatic approach: surgical approaches and perioperative outcomes
}

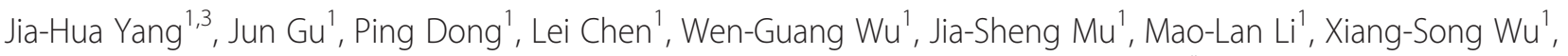 \\ Yang-Lu Zhao' ${ }^{2}$ Lin Zhang ${ }^{1}$, Hao Weng ${ }^{1}$, Qian Ding ${ }^{1}$, Qi-Chen Ding ${ }^{1}$ and Ying-Bin Liu ${ }^{1 *}$
}

\begin{abstract}
Background: How to resect the caudate lobe safely is a major challenge to current liver surgery which requires further study.

Methods: Nine cases ( 6 hepatic cell carcinoma, 2 cavernous hemangioma and 1 intrahepatic cholangiocacinoma) were performed using the anterior transhepatic approach in the isolated complete caudate lobe resection. During the operation, we used the following techniques: the intraoperative routine use of Peng's multifunction operative dissector (PMOD), inflow and outflow of hepatic blood control, low central venous pressure and selective use of liver hanging maneuver.
\end{abstract}

Results: There were no perioperative deaths observed after the operation. The median operating time was $230 \pm$ 43.6 minutes, the median intraoperative blood loss was $606.6 \pm 266.3 \mathrm{ml}$ and the median length of postoperative hospital stay was $12.6 \pm 2.9$ days. The incidence of complications was $22.22 \%(2 / 9)$.

Conclusion: PMOD and "curettage and aspiration" technique can be of great help of in the dissection of vessels and parenchyma, clearly making caudate lobe resection safer, easier and faster.

\section{Background}

The caudate lobe, which is generally divided into three regions: the left Spiegel's portion, the process portion, and the paracaval portion, is located in a complex anatomical position, deep behind the confluence of the main hepatic veins, porta hepatis and inferior vena [1]. In other words, it is surrounded by three portae hepatis. The blood supply and biliary drainage of the caudate lobe come from both the left and the right portal triads, called the caudate portal triads (CPT). However, the number of triads may vary. Venous drainage (short hepatic vein) occurs along its posterior aspect directly into the inferior vena cava (IVC) through several small branches of variable size and location. Biliary drainage includes small tributaries to the right but occurs predominantly through the left hepatic duct.

\footnotetext{
* Correspondence: liuybphd@126.com

${ }^{1}$ Department of General Surgery, Xinhua Hospital, School of Medicine,

Shanghai Jiaotong University, 1665 Kongjiang Road, Shanghai 200092, China

Full list of author information is available at the end of the article
}

Due to the deep location and position between the major vascular structures, the caudate lobe has been always considered a forbidden area for hepatic surgery, and its resection is always a challenge for hepatobiliary surgeons. However, with solid knowledge of the anatomical relationship, mastery of the appropriate surgical instrument and thorough experience of performing the operation, the caudate lobectomy can be carried out safely. Isolated complete caudate lobectomy is the most difficult and most complex of the various methods of caudate lobe resection [2]. The anterior transhepatic approach for isolated complete caudate lobe resection has been carried out in our department. In this article, it is proved to be safe, effective and clinically feasible using the curettage and aspiration technique (CADT) and a special instrument, Peng's multifunction operative dissector (PMOD) (Figure 1). 


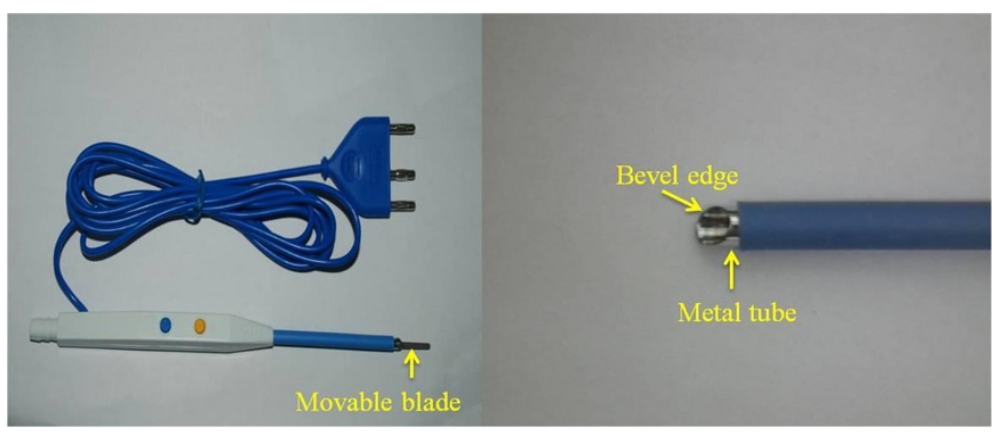

Figure 1 Peng's multifunction operative dissector (PMOD) and curettage and aspiration dissection technique (CADT).

\section{Case Presentation \\ Patients}

Five male and four female patients with a median age of 57 years received isolated hepatic caudate lobectomy between January 2005 and December 2011. Postoperative pathology results identified six patients with hepatocellular carcinoma, two with cavernous hemangioma, and one with intrahepatic cholangiocacinoma. All patients' liver function was in Child-Pugh class A.

\section{Surgical procedures}

Isolated complete caudate lobectomy using the anterior transhepatic approach included six steps:

1. Liver mobilization: the ligamentum teres hepatis was ligated and the falciform ligament was incised from the anterior abdominal wall to the front of the suprahepatic inferior vena cava (SIVC). Then the roots of the major hepatic veins were exposed. After the lesser omentum was incised, both the left Spiegel lobe and the left side of the SIVC would be exposed. The incision was made to the right, and the right coronary ligament, right triangular ligament, and hepatorenal ligament were dissected.

2. Blood flow control: the retroperitoneum overlying the infrahepatic inferior vena cava (IIVC) was opened at a position right of the IIVC and 1 to $2 \mathrm{~cm}$

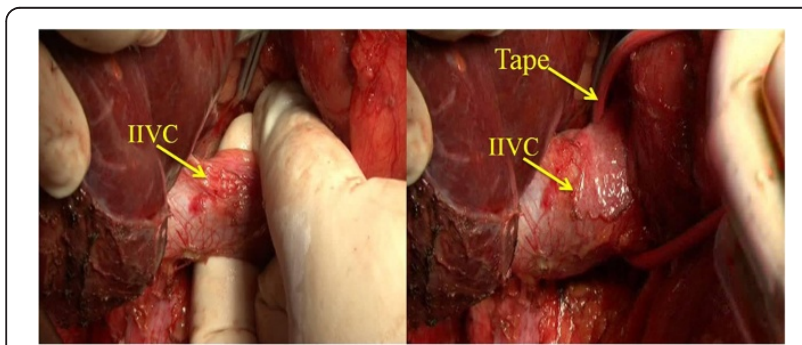

Figure 2 Opening the retroperitoneum. The retroperitoneum overlying the infrahepatic inferior vena cava (IIVC) is opened (left). The IIVC is taped (right). above the right renal vein. Then the surgeon passed his left index finger behind the IIVC to the left side and guided a tape to encircle the IIVC (Figure 2). The SIVC was dissected from its posterior structure and a clamp was passed through the tunnel behind it toward the left side, then the surgeon encircled the SIVC with tape (Figure 3). Finally, the hepatoduodenal ligament was mobilized and encircled with tape (Figure 4).

3. Short hepatic vein ligation: three to five thick, short hepatic veins were separated in this process. Blunt dissection was used to develop the tunnel before a tape was passed through (Figure 5).

4. Liver-splitting anterior approach: the interlobar plane had been split before the anterior surface of the paracaval portion and the hilar plate were explored.

5. Caudate portal triad ligation: there were three to five caudate portal triads branching from the left and right hepatic pedicle junction into the caudate lobe.

6. Detachment of the caudate lobe from the eighboring liver parenchyma. We used PMOD to transect the liver parenchyma by means of the CADT when intermittent inflow was occlusive (Figures 6 and 7). Tapes were used to encircle the IIVC and hepatic pedicle. The time limit was ten minutes each time with reperfusion for two minutes.

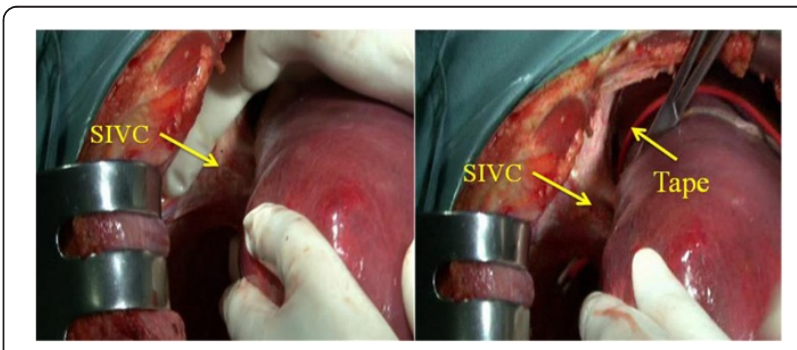

Figure 3 The tunnel (arrow) was posterior to the suprahepatic inferior vena cava SIVC. The position of the tunnel is shown (left). A clamp was passed beneath in the channel toward the left side, then a tape was guided to tap the SIVC. 


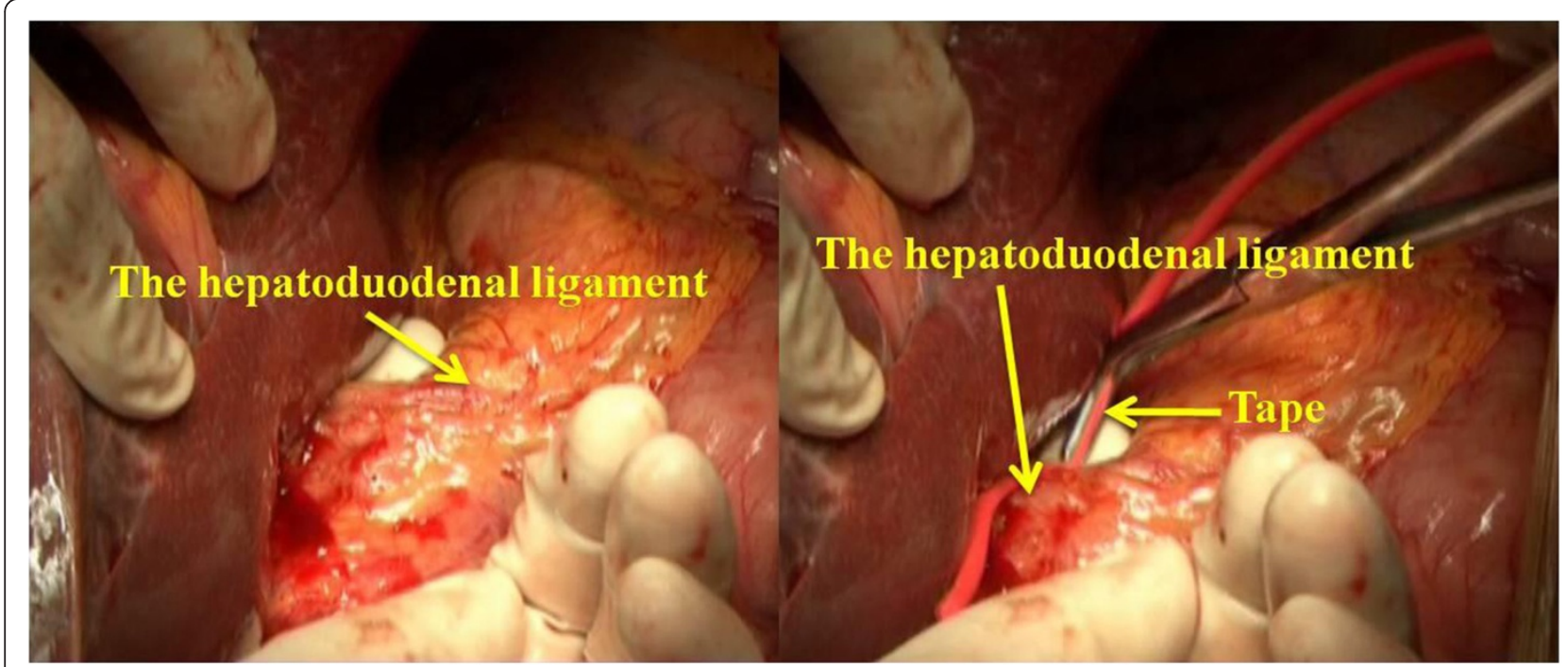

Figure 4 Mobilizing the hepatoduodenal ligament and encircling it with tape.

\section{Results}

There were 6 cases with hepatocarcinoma (HCC) associated with liver cirrhosis, 2 cases with large cavernous hemangioma and 1 case with large intrahepatic cholangiocacinoma. All patients had liver function in Child-Pugh A class. Liver hanging maneuver was performed successfully in 8 patients. The median tumor size was $6.2 \pm 2.7 \mathrm{~cm}$ (range 2.1-10.5 cm). The median operating time was $230 \pm 43.6 \mathrm{~min}$ (range 170-300 min). The median intraoperative blood loss was $606.6 \pm 266.3 \mathrm{ml}$ (range 350-1200 ml). All patients received R0 resections. The median length of postoperative hospital stay was $12.6 \pm 2.9$ days. Two complications were observed in two patients, including ascits in one patient and bile leakage in another. Both of them were successfully managed medically. There were no perioperative deaths (Table 1).

\section{Discussion}

Removal of excess liver tissue is not allowed in the process of the hepatic caudate lobectomy, because in China $85 \%$ of cases of hepatocarcinoma (HCC) are complicated by cirrhosis. So, isolated resection of the hepatic lobe plays an irreplaceable role in surgical treatment of hepatic tumor. Both left- and right-side approaches are used to resect the caudate lobe when the tumor is small. But when the tumor is large and compresses major hepatic veins, or when cirrhosis is very serious, the

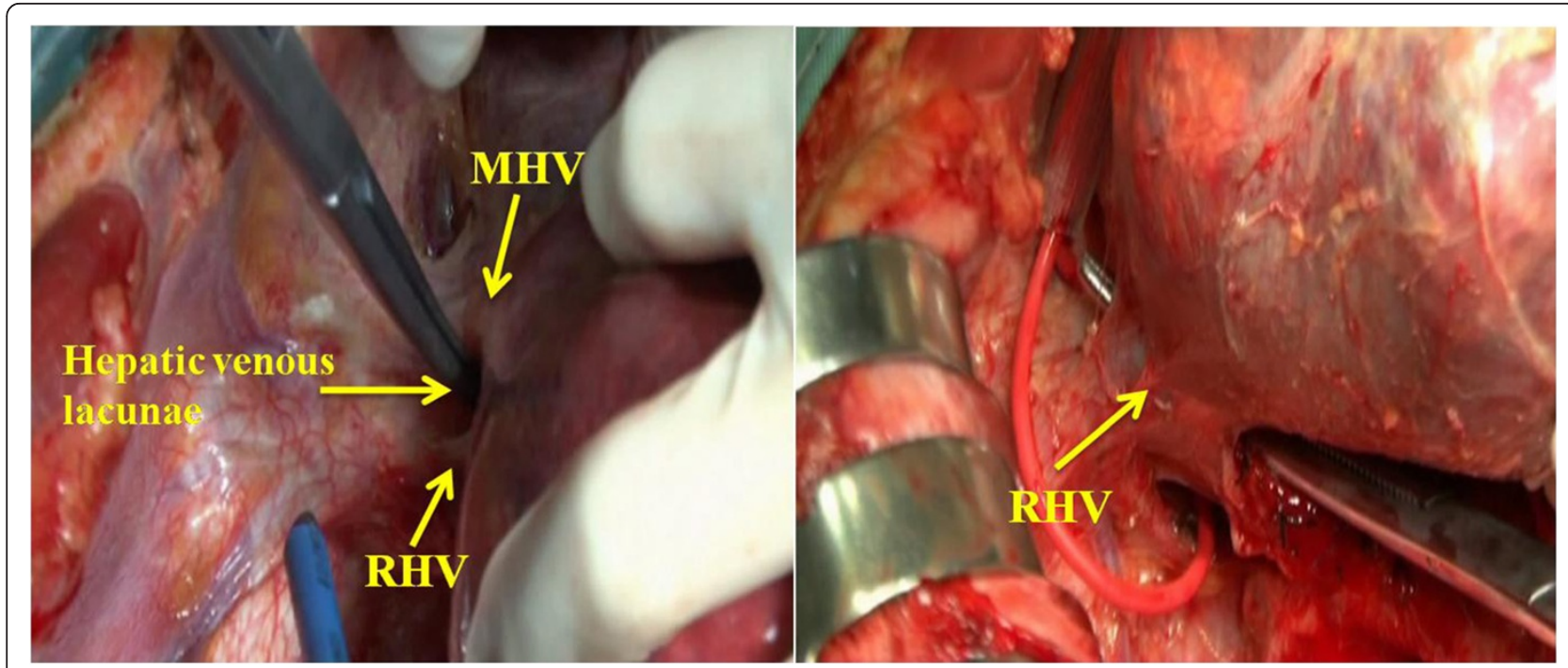

Figure 5 Blunt dissection was used to develop the tunnel before a tape was passed through. 


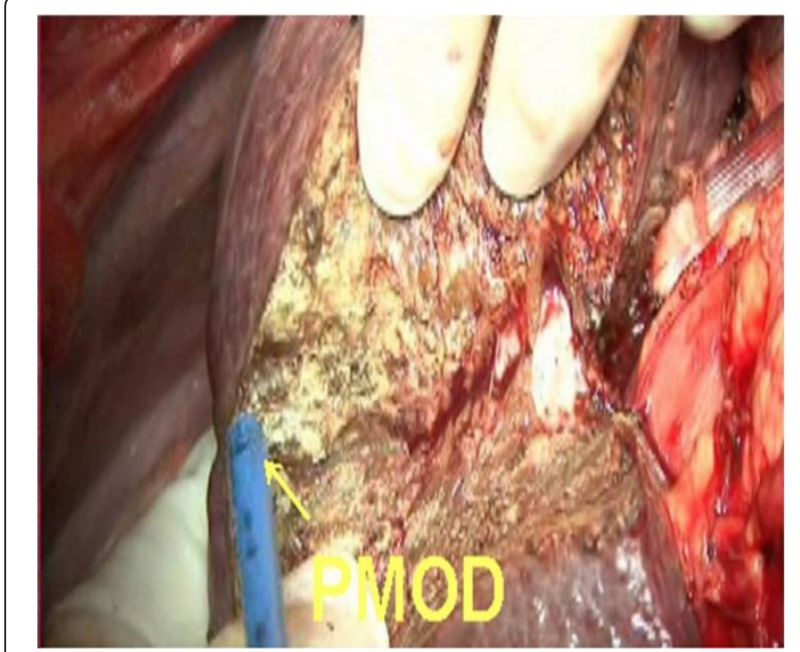

Figure 6 The parenchymal has been transected by Peng's multifunction operative dissector (PMOD) and the curettage and aspiration dissection technique (CADT).

above methods cannot be performed because of possible injury to the major hepatic veins [3]. Based on these circumstances, the anterior transhepatic approach is the best choice for isolated complete caudate lobectomy, because it maximizes the exposure of the operative field, and minimizes the operative risks.

Liver resection may be complex due to prolonged operating times and intraoperative bleeding, especially during the separation of the hepatic parenchyma and the resection of lesions close to major hepatic veins, in which unpredictable hemorrhage can be life-threatening [4,5]. PMOD combines four different functions in one: electro-cutting, electro-coagulating, curetting and aspirating [6]. The 40 to $60 \mathrm{HZ}$ power would be sufficient for separation of the hepatic ligament, and the maximum power of $120 \mathrm{HZ}$ can be used for transection of the hepatic parenchyma [7]. We mainly use it to curette on the liver incision line for parenchymal transection. When large vessels are seen, curettage is applied to separate the parenchyma from the vessels in the same layer. Then the vessels can be ligated and dissected in direct view. Therefore, sudden and massive bleeding rarely occurs. Both operating times and hemorrhage are thereby reduced remarkably to make the operation safer. Thus, in our study, the mean operating time and blood loss were 232.2 minutes and $606.6 \mathrm{ml}$ respectively, which are lower than in other reports [8-12].

It has been widely proposed to use hepatic vascular control in hepatic caudate lobectomy [5], but some authors regard it as unnecessary [13]. Low, central venous pressure (CVP) can decrease the pressure in the hepatic veins and hepatic sinusoid, thereby reducing bleeding from these locations [14]. If tapes or blood control in the IIVC are used, CVP could be decreased by 4 to $6 \mathrm{~cm} \mathrm{H}_{2} \mathrm{O}\left(1 \mathrm{~cm} \mathrm{H}_{2} \mathrm{O}=0.098 \mathrm{kPa}\right)$ [8]. It was also reported that during surgery, the blood control in the IIVC had the same effect as hypotensive anesthesia in reducing CVP [15]. If the hepatic pedicle and the IIVC are both encircled, bleeding of the hepatic cutting surface will be reduced significantly. If the tumor is so large that it is difficult to implement the IVC blood control for precaution, the surgeon should not try to use tape to encircle the SIVC. However, tumors adhering to the IIVC need to be mobilized to free the IVC to the renal vein, then the tape can encircle the SIVC.

Blunt dissection is used to make the tunnel before the tape is pulled through [16]. A hemostatic plate may be placed on the surface of liver parenchyma if needed. In hepatectomy, the tape is pulled up to create an interspace between liver parenchyma and the IVC so the IVC can be protected during transection. With this method, we can separate the liver in the possible shortest time,

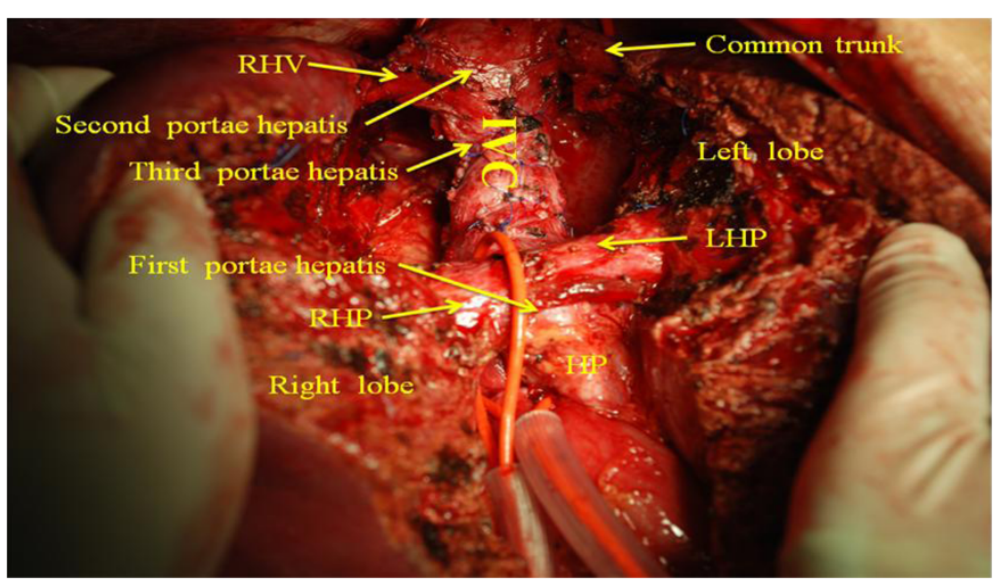

Figure 7 The liver was split into two halves through the midplane, thus the caudate lobe as well as the first, second, third portae hepatis were fully exposed. 
Table 1 General data from the nine patients

\begin{tabular}{|c|c|c|c|c|c|c|}
\hline Patient & $\begin{array}{c}\text { Age/ } \\
\text { sex }\end{array}$ & $\begin{array}{l}\text { Tumor } \\
\text { size } \\
(\mathrm{cm})\end{array}$ & Pathology & $\begin{array}{c}\text { Operating } \\
\text { time } \\
\text { (minutes) }\end{array}$ & $\begin{array}{l}\text { Blood loss } \\
(\mathrm{ml})\end{array}$ & $\begin{array}{c}\text { Postoperative } \\
\text { hospital stay (days) }\end{array}$ \\
\hline 1 & $45 / \mathrm{M}$ & 5.2 & Hepatocarcinoma & 210 & 600 & 14 \\
\hline 2 & $48 / F$ & 7.9 & Hemangioma & 255 & 500 & 12 \\
\hline 3 & $55 / F$ & 6.2 & Hepatocarcinoma & 240 & 800 & 10 \\
\hline 4 & $52 / \mathrm{M}$ & 7.4 & Hholangiocacinoma & 270 & 1,200 & 8 \\
\hline 5 & $60 / F$ & 2.1 & Hepatocarcinoma & 170 & 450 & 18 \\
\hline 6 & $65 / M$ & 10.5 & Hemangioma & 260 & 700 & 11 \\
\hline 7 & $62 / M$ & 3.2 & Hepatocarcinoma & 205 & 400 & 13 \\
\hline 8 & $70 / F$ & 8.6 & Hepatocarcinoma & 300 & 450 & 12 \\
\hline 9 & $56 / \mathrm{M}$ & 4.3 & Hepatocarcinoma & 180 & 350 & 15 \\
\hline All patients (mean \pm SD) & $57 \pm 8.1$ & $6.2 \pm 2.7$ & NA & $230 \pm 43.6$ & $606.6 \pm 266.3$ & $12.56 \pm 2.9$ \\
\hline
\end{tabular}

Quantitative data are presented as absolute values for individual patients unless stated otherwise. $M$, Male; $F$, Female; NA, Not applicable.

and the surgical risk will be reduced to a minimum. Only when the IVC is invaded by the tumor can the liver-hanging maneuver not be performed.

\section{Conclusions}

The anterior transhepatic approach in isolated complete caudate lobectomy is a curative procedure for the tumor located in caudate 1obe, especially suitable for cases with large tumor, cirrhosis and IVC invasion. The application of anterior approach for isolated caudate lobectomy can converse the results of certain kind of caudate lobe tumors from non-resectable to respectable due to widening the indication. The intraoperative routine use of PMOD, application of inflow and outflow of hepatic vascular control, low central venous pressure and selective use of liver hanging maneuver together make the anterior transhepatic approach for isolated complete caudate lobectomy safer and easier.

\section{Consent}

Written informed consent was obtained from the patients for publication of this report and any accompanying images.

\section{Abbreviations}

CADT: Curettation and aspiration technique; CPT: Caudate portal triads; CVP: Central venous pressure; HCC: Hepatocarcinoma; IVVC: Infrahepatic inferior vena cava; IVC: Inferior vena cava; PMOD: Peng's multifunction operative dissector; SIVC: Suprahepatic inferior vena cava.

\section{Competing interests}

The authors declare that they have no competing interests.

\section{Authors' contributions}

YJH, GJ, DP, CL, WWG, MJS, LML, WXS, ZYL, ZL, WH, and DQ designed and conducted the study, analyzed the data, and helped to write the manuscript. LYB is the principal investigator, and revised and edited the manuscript. All authors approved the final manuscript.

\section{Author details}

${ }^{1}$ Department of General Surgery, Xinhua Hospital, School of Medicine, Shanghai Jiaotong University, 1665 Kongjiang Road, Shanghai 200092, China. ${ }^{2}$ Shanghai Jiaotong University Xinhua Clinical Medical School, 1665 Kongjiang Road, Shanghai 200092, China. ${ }^{3}$ Department of General Surgery, Putuo Hospital, Shanghai University of Traditional Chinese Medicine, Shanghai, China.

Received: 21 December 2012 Accepted: 5 August 2013

Published: 16 August 2013

\section{References}

1. Kumon M: Anatomy of the caudate lobe with special reference to portal vein and bile duct. Acta Hepatol Jpn 1985, 26:1193-1199.

2. Peng SY, Li JT, Mou YP, Liu YB, Wu YL, Fang HQ, Cao LP, Chen L, Cai XJ, Peng $\mathrm{CH}$ : Different approaches to caudate lobectomy with "curettage and aspiration" technique using a special instrument PMOD: a report of 76 cases. World J Gastroenterol 2003, 9:2169-2173.

3. Wang Y, Zhang LY, Yuan L, Sun FY, Wei TG: Isolated caudate lobe resection for hepatic tumor: surgical approaches and perioperative outcomes. Am J Surg 2010, 200:346-351.

4. Popescu I, Ciurea S, Romanescu D, Boros M: Isolated resection of the caudate lobe: indications, technique and results. Hepatogastroenterology 2008, 55:831-835.

5. Chaib E, Ribeiro MA Jr, Silva Fde S, Saad WA, Cecconello I: Surgical approach for hepatic caudate lobectomy: Review of 401 cases. J Am Coll Surg 2007, 204:118-127.

6. Wenguang W, Xuefeng W, Zhiping Z, Xiangsong W, Jianwei W, Songgang L, Yingbin L: Three-step method for lymphadenectomy in gastric cancer surgery: a single institution experience of 120 patients. J Am Coll 2011, 212:200-208.

7. Peng SY, Li JT: "Curettage and aspiration dissection technique" using PMOD for liver resection. HPB 2008, 10:285-288.

8. Wahab MA, Fathy O, Elhanafy E, Atif E, Sultan AM, Salah T, Elshoubary M, Anwar N, Sultan A: Caudate lobe resection for hepatocellular carcinoma. Hepatogastroenterology 2011, 58:1904-1908.

9. Terayama N, Miyayama S, Tatsu H, Yamamoto T, Toya D, Tanaka N, Mitsui T, Miura S, Fujisawa M, Kifune K, Matsui O, Takashima T: Subsegmental transcatheter arterial embolization for hepatocellular carcinoma in the caudate lobe. J Vasc Interv Radiol 1998, 9:501-508.

10. Takayama T, Tanaka T, Higaki T, Katou K, Teshima Y, Makuuchi M: High dorsal resection of the liver. J Am Coll Surg 1994, 179:72-75.

11. Yamamoto J, Takayama T, Kosuge T, Yoshida J, Shimada K, Yamasaki S, Hasegawa $\mathrm{H}$ : An isolated caudate lobectomy by the transhepatic approach for hepatocellular carcinoma in cirrhotic liver. Surgery 1992, 111:699-702.

12. Yanaga K, Matsumata T, Hayashi H, Shimada M, Urata K, Sugimachi K: Isolated hepatic caudate lobectomy. Surgery 1994, 115:757-761. 
13. Sarmiento JM, Que FG, Nagorney DM: Surgical outcomes of isolated caudate lobe resection: a single series of 19 patients. Surgery 2002, 132:697-709.

14. Jones RM, Moulton CE, Hardy KJ: Central venous pressure and its effect on blood loss during liver resection. Br J Surg 1998, 85:1058-1060.

15. Melendez JA, Arslan V, Fischer ME, Wuest $D$, Jarnagin WR, Fong $Y$,

Blumgart LH: Perioperative outcomes of major hepatic resections under low central venous pressure anesthesia: blood loss, blood transfusion, and the risk of postoperative renal dysfunction. J Am Coll Surg 1998, 187:620-625.

16. Belghiti J, Guevara OA, Noun R, Saldinger PF, Kianmanesh R: Liver hanging maneuver: a safe approach to right hepatectomy without liver mobilization. J Am Coll Surg 2001, 193:109-111.

\section{doi:10.1186/1477-7819-11-197}

Cite this article as: Yang et al:: Isolated complete caudate lobectomy for hepatic tumor of the anterior transhepatic approach: surgical approaches and perioperative outcomes. World Journal of Surgical Oncology 2013 11:197.

\section{Submit your next manuscript to BioMed Central and take full advantage of:}

- Convenient online submission

- Thorough peer review

- No space constraints or color figure charges

- Immediate publication on acceptance

- Inclusion in PubMed, CAS, Scopus and Google Scholar

- Research which is freely available for redistribution 